\title{
Brief Compassion-Focused Imagery Dampens Physiological Pain Responses
}

\author{
Frances A. Maratos ${ }^{1}$ (D) . David Sheffield ${ }^{1}$
}

Published online: 3 September 2020

(C) The Author(s) 2020

\begin{abstract}
Objectives Affiliative processes are postulated to improve pain coping. Comparatively, compassion-focused imagery (CFI) also stimulates affiliate affect systems with a burgeoning behavioural, cognitive and physiological evidence base. Thus, the purpose of the present research was to investigate if engaging in brief CFI could improve pain coping.

Methods Utilising a randomised repeated measures crossover design, 37 participants were subjected to experimental pain (cold pressor) following counter-balanced engagement with CFI or control imagery, 1 week apart. Salivary alpha-amylase (sAA) and questionnaire measures of emotional responding were taken: at baseline, following introduction to the imagery condition (anticipation), and immediately after the cold pressor pain task (actual).

Results Participants exhibited increases in SAA levels in response to pain following control imagery but, no such changes were observed following CFI (i.e. there was a significant time-by-condition interaction). Pain tolerance (the length of time participants immersed their hands in the cold pressor) did not differ by imagery condition. However, sAA responses to actual pain predicted decreased pain tolerance in the CFI condition. Additionally, anticipatory sAA response predicted increased pain tolerance across both conditions. None of the emotional measures of well-being differed by imagery condition, nor by condition over time.

Conclusions These data demonstrate that using CFI can curtail a physiological stress response to pain, as indicated by increases in sAA in the control imagery condition only, following pain; pain tolerance was not influenced by CFI. Compassion-based approaches may therefore help people cope with the stress associated with pain.
\end{abstract}

Keywords Compassion $\cdot$ Acute pain $\cdot$ Pain anticipation $\cdot$ Pain tolerance $\cdot$ Salivary alpha-amylase $\cdot$ Nature

There is growing evidence that focusing on the cultivation of compassion-based emotions has important effects on mental states and well-being (Hofmann et al. 2011; Maratos et al. 2019). Research demonstrates that compassion-focused interventions are effective in both clinical and non-clinical populations (Kirby et al. 2017b). The effectiveness of such interventions is often argued to reflect their empirical bases, including that compassion-focused interventions are derived from an understanding of evolutionary and functional analysis of basic motivational systems. To expand, in the compassion approach originated by Gilbert (see Gilbert 2014, for review), it is argued that the brain is highly evolved for social

Frances A. Maratos

f.maratos@derby.ac.uk

1 Human Sciences Research Centre, University of Derby, Kedleston Road, Derby DE22 1GB, UK processes. Central to this, and supported by neurophysiological research (e.g. Longe et al. 2010; but see also Kim et al. 2020a), is the idea of three affect systems within the brain. These are the threat, drive and soothing systems.

The threat-protection system is related to fight-flight tendencies and emotions of anger and anxiety related to sympathetic nervous system activity (see Maratos and Pessoa 2019, for review). The drive system is related to high arousal positive 'approach' emotions such as pleasure and excitement (Depue and Morrone-Strupinsky 2005). The soothing system is related to those emotions not associated with 'threat' or 'doing' (Gilbert 2017); that is, those emotions associated with a state of quiescence, including feeling safe, peaceful and contented. The soothing system also includes emotions related to caring and affiliative behaviours that have been key to mammalian evolution. Importantly, compassion-focused interventions are hypothesised to be linked to the soothing/ affiliative system. It is opined that 'training' the soothing system (using compassion-focused interventions) allows for 
better regulation of threat and drive systems linked to sympathetic nervous system activation (Duarte et al. 2015). Therefore, it is argued that compassion-based interventions do not only impact upon psychological health, but also physiological health (Kim et al. 2020b; Singer and Bolz 2013).

In accord with this, research has demonstrated that compassion-focused interventions stimulate oxytocin, endorphin and regulate sympathetic/parasympathetic activity (for review see Kirby et al. 2017a). For example, Arch et al. (2014) investigated if brief compassion training could diminish physiological stress. Once individuals had been trained in self-compassion or a control intervention, they took measures of both cortisol and salivary alpha-amylase (sAA) pre and post a social stress test. Cortisol was their measure of hypothalamic-pituitary-adrenal (HPA) axis activity (often associated with chronic stress responses; see Sapolsky et al. 1986); and sAA their measure of acute sympathetic nervous system activity (see van Stegeren et al. 2006). They found that brief self-compassion training dampened/flattened sAA level increases - but not cortisol — and thus concluded that this brief training impacts the fast-acting sympathetic system stress response, implicated in defensive reactions. Further research has demonstrated that receiving oxytocin can increase the ease at which individuals can engage in compassion-focused imagery (CFI). CFI is a brief visualisation technique often used within compassion-focused interventions (see Rockliff et al. 2011). Thus, compassion-based interventions appear to allow for a state of quiescence, or sympathetic/parasympathetic activity regulation, achieved via (i) dampening of the threat-focused affect system and/or (ii) stimulation of the affiliative affect system.

Interestingly, activation of the affiliative affect system may also be related to pain coping and pain responses. That is, psychological and behavioural research has demonstrated that social support, whether real or imagined, can increase pain tolerance and psychological pain coping (Brown et al. 2003; Cano and Williams 2010; Jackson et al. 2005; Master et al. 2009). Brown et al. (2003) found that participants who received support had greater pain tolerance to cold pressor than those who completed the task alone or with no support. Further, Master et al. (2009) reported that viewing a loved one's photograph lowered pain responses to thermal pain stimuli compared with viewing objects, suggesting enacting mental representations of loved ones may be sufficient to alter pain responses. Thus, it may be that compassion-based interventions can be used to address pain coping.

Gooding et al. (2020) further argue that in individuals who experience persistent pain, the threat system may be dysregulated (i.e. over-activated). As such, they state compassionbased interventions may help patients with pain better regulate their emotions, allowing for increased pain coping. Consistent with this, in populations with persistent pain, self-compassion has been found to predict pain disability and adjustment to pain (Purdie and Morley 2016; Wren et al. 2012). Penlington (2019) further reported that a compassionfocused intervention for persistent pain was associated with reduced pain distress, intensity, anxiety and depression, and increased self-efficacy.

Taken as a whole, such research demonstrates the potential buffering effects of affiliative/compassion-based practices when coping with pain. Therefore, the aim of the present study was to explore if brief CFI could improve responses to acute painful stimuli. Specifically, whether engaging in CFI could increase pain tolerance and/or decrease accompanying stress physiology, i.e. salivary alpha-amylase (Nater and Rohleder 2009) as well as impact upon psychological indicators of positive affect, associated with threat, drive and soothing emotions. We used sAA as our measure of physiological stress, because $\mathrm{SAA}$ (i) has been implicated in rapid/acute sympathetic nervous system activation (Arch et al. 2014; van Stegeren et al. 2006) and (ii) correlates positively with ratings of acute pain intensity and unpleasantness (Wittwer et al. 2016). As CFIs have also been found to be effective in reducing fear and anticipatory anxiety (Harwood and Kocovski 2017), a second aim was to explore the effects of CFI on pain anticipation. To this end, participants practiced and then engaged in control (nature) imagery and CFI whilst subjected to painful stimulation (cold pressor task). To control for order-effects, crossover effects and diurnal variability in sAA, participants took part in the two imagery conditions 7 days apart, but at the same time of day, and order of imagery conditions was counter-balanced.

\section{Methods}

\section{Participants}

Sample size was calculated based on the medium effect size of Arch et al. (2014). To obtain an interaction effect for this repeated measures design with a medium effect size $(0.25)$ and acceptable power (i.e. 0.8; with alpha set at 0.05 , twotailed), the calculated sample size required was 34 . Thirtyseven participants were recruited to the research from the local University community (i.e. staff and students). Exclusion criteria included pregnancy, thrombosis (blood clot), heart problems, chronic pain, peripheral neuropathy (loss or impairment of sensation in hands/feet), circulatory disorders including Reynaud's disease and unmedicated high or low blood pressure. Following screening, data from three participants were removed due to extreme scoring on the baseline selfcriticism measure ( $>40)$; as self-criticism has been found to affect responses to CFI (Duarte et al. 2015; Rockliff et al. 2011) and implicated in responding to pain (Gregory et al. 2017). This resulted in a final sample of 34 participants (16 male, 18 female, mean age 25.6 years, age range 19-43). 
All participants gave informed written consent to participate in the study, which received local Ethics Committee approval. Participants who completed all aspects of the experiment (i.e. both conditions) received vouchers to the value of $£ 10$ on completion.

\section{Procedure}

We employed a repeated measures crossover design (AB/ BA), with imagery type (CFI vs. control) as the withinsubjects counter-balanced variable. Data for each participant in both conditions was collected at the same time of day separated by seven days. The choice of a randomised crossover repeated design enabled the minimisation of the influence of individual differences and extraneous variables to most clearly examine the effects of the intervention (Mills et al. 2009); checks for carryover effects were conducted. Saliva samples for alpha-amylase were taken at three time points within each imagery condition.

The CFI and control imagery interventions consisted of three phases. In phase 1, participants signed the consent forms, were briefed as to the cold pressor task and completed the baseline measures (i.e. FSCSR, TPAS, PANAS). Mid-way through this phase, at approximately $7.5 \mathrm{~min}$, the first sAA measurement was taken (i.e. 'baseline' level). In phase 2, all participants listened to a three-minute relaxation imagery (focused on grounded breathing) and then practiced the relevant experimental imagery ( $7 \mathrm{~min}$; either CFI or control, counterbalanced across participants). Immediately after this practice, the second sAA sample was taken followed by the state selfreport measures (i.e. TPAS, PANAS). In the final phase, participants were asked to immerse their non-dominant hand up to their wrist in $3{ }^{\circ} \mathrm{C}$ water. The relevant audio imagery was again played to participants as they immersed their hand into the water. Participants were asked to focus/engage with the audio imagery whilst their hand was immersed, but to remove their hand from the cold water when they could tolerate the pain no longer (up to a maximum of $5 \mathrm{~min}$ ). Upon withdrawal of their hand from the water, the third and final sAA sample was taken followed again by the two state self-report measures (i.e. TPAS, PANAS).

\section{Compassion-Focused Imagery Task}

In the CFI condition participants listened to a recorded outline of the imagery task, a definition of compassion, an explanation of what was meant by 'mental imagery', and advice on how to deal with one's mind wandering (i.e. not to worry, but simply guide one's mind back to the imagery). The CFI involved participants generating visual images of a deeply compassionate person/being. Using a standard recording, the participants were asked to imagine being the recipient of compassion and feelings of warmth, understanding, and care, emanating from this image to them. The recording guided each participant through the CFI, with verbal prompts at 45$\mathrm{s}$ intervals, focusing on compassionate qualities-e.g. 'focus on the wisdom and understanding that is there for you; imagine being understood and completely accepted; focus on the great warmth and kindness that permeates the whole image and is directed at you'. Transcripts and imagery instructions were the same as that used by Rockliff et al. (2008).

\section{Control Imagery Task}

In the control imagery condition, a similar procedure was adopted, but with the exception that the recorded imagery task involved a scenario where individuals were asked to engage in imagery concerned with taking a stroll through the countryside/some woods. Importantly, this imagery task was matched to the CFI task on a number of key variables. It was of the same length as the CFI task, contained the same number of verbal prompts at similar time intervals and used the same actress as in the CFI audio recording. Transcripts and imagery instructions were the same as that used by Duarte et al. (2015).

The use of this task was to ensure that participants engaged in a similar type of intervention (i.e. imagery) of matched duration, but one that was not associated with any particular physiological responses, nor any particular emotions.

\section{Measures}

\section{Alpha-Amylase Measurement}

sAA measurement was obtained via saliva samples (Sarstedt Ltd.; Leicester, UK). For each sample, participants were asked to rinse their mouths with cold water ( $3 \mathrm{~min}$ ), and then place rolling cotton under their tongue until saturated (about $3 \mathrm{~min}$ ). All samples were then immediately frozen $\left(\right.$ at $\left.-70^{\circ} \mathrm{C}\right)$ before being assayed for alpha-amylase by Obsidian Research Ltd. (Port Talbot, UK) using an enzyme-linked immunosorbent.

To minimise the effects of circadian oscillations of alphaamylase (Rohleder and Nater 2009), all experimental sessions were conducted between 2 and 7 PM. Additionally, to reduce confounding factors shown to affect physiological dependent measures (Nater et al. 2007), before each experimental session, participants were asked to refrain from a number of behaviours including: excessive physical activity for the preceding $48 \mathrm{~h}$ period; sporting activities for the preceding $24 \mathrm{~h}$; alcohol intake for the preceding $18 \mathrm{~h}$ period; glucose/ caffeine intake and chewing gum on the day of the study; and tooth-brushing, eating or drinking (except water) for the preceding $60 \mathrm{~min}$ period. 


\section{Pain Tolerance}

Pain tolerance was recorded as the length of time (in seconds) that participants immersed their hands in the cold pressor tank up to $300 \mathrm{~s}$; at $300 \mathrm{~s}$ participants were instructed to remove their hands and a time of $300 \mathrm{~s}$ was recorded. The cold pressor consisted of a 12-1 tank of circulating water and ice; the temperature was thermostatically controlled to ensure that the water remained at $3{ }^{\circ} \mathrm{C}$ (Mitchell et al. 2004).

\section{Forms of Self-Criticism/Self-Reassuring Scale (FSCRS; Gilbert et al. 2004)}

This 22-item trait scale assesses participants' thoughts and feelings about themselves during a perceived failure. Two subscales measure forms of self-criticizing (inadequate self and hated self) and one subscale measures tendencies to be reassuring to the self(reassured self). Items are rated on a fivepoint Likert scale. Cronbach's alphas are 0.90 for inadequate self, 0.86 for hated self and 0.86 for reassured self. We used this measure to screen for high self-criticism. Validity has been supported through correlation with further measures of self-criticism and depression in the expected directions (i.e. positive for inadequate self and hated self, negative for reassured self; Gilbert et al. 2004).

\section{Types of Positive Affect Scale (TPAS; Gilbert et al. 2008)}

This 12-item state scale measures activated, relaxed and secure/safe positive affect. Participants rate (i) how characteristic each feeling (i.e. affective word such as 'calm', 'secure', 'content') is for them, as well as (ii) their enjoyment from such a feeling state, on a five-point Likert scale. Cronbach's alphas are 0.83 for the activated and relaxed subscales and 0.73 for the safe subscale. We used this measure to explore quiescence (i.e. psychological activation of the soothing/affiliation system) as a consequence of the imagery intervention. Previously, Rockliff et al. (2011) have demonstrated brief CFI to result in increased 'relaxed' positive affect and 'decreased' activated positive affect.

\section{Positive Affect and Negative Affect Schedule (PANAS; Watson et al. 1988)}

This 20-item mood scale provides brief measures of positive and negative affect (10 items each respectively). Respondents rate the extent to which they have experienced each particular emotion within a specific time period (right now), using a five-point Likert scale. Cronbach's alphas range from 0.86 to 0.90 for the positive affect scale, and 0.84 to 0.87 for the negative affect scale. We used this measure to explore affect (i.e. downregulation of threat and upregulation of positive affect) as a consequence of the imagery intervention. The
PANAS is a widely used measure of positive and negative affect, with reliability and validity supported through correlation with common measures of psychopathology across a range of populations (see, for example, Serafini et al. 2016).

\section{Fidelity of Imagery Measure}

In order to assess ability to engage with the CFI and control imagery interventions, participants were asked to complete five ten-point Likert scale items post the cold pressor task. These asked: how easy it was to experience the imagery (q1), whether they wanted to resist the imagery (q2), how hard they tried to create a visual image (q3), how clearly could they see their image (q4) and how tense they felt at that moment (q5). These questions were included in order to establish any differences in fidelity of the imagery tasks, which would have implications for interpreting results.

\section{Data Analyses}

\section{sAA Data Processing, Screening \& Analysis}

To accurately analyse the sAA data, we computed delta scores by subtracting the baseline phase from each condition as recommended by Rohleder and Nater (2009). This data was analysed for normality of distribution using Shapiro-Wilk tests alongside plots to examine skewness and kurtosis scores; these revealed delta SAA values to be normally distributed. Next, checks for carryover effects were conducted by including order terms in the initial ANOVA (Chin and Lee, 2008). Consequently, a repeated measures ANCOVA was conducted with imagery condition (CFI or control) and time (anticipation, pain) as the within-subjects IV, age (Maratos et al. 2017) and gender served as the covariates, and delta score as the dependent variable.

\section{Pain Tolerance}

To assess whether there were differences between the CFI and control imagery conditions in the amount of time individuals kept their hand immersed, a Cox proportional hazards regression model was conducted. Cox regression (or proportional hazards regression) is a method for investigating the effect of variables upon the time a specified event takes to happen and has previously been used to examine the effects of an intervention on cold pressor tolerance (Smith and Norman, 2017). It permits tolerance time and 'completion' to be analysed together and is statistically robust. In the current study, hand withdrawal was defined as an event and individuals who endured the full $5 \mathrm{~min}$ were treated as censored in the analysis. 


\section{Emotional Indicator Data}

To assess change in our state measures during the interventions a repeated measures MANOVA was conducted, with time (i.e. baseline, anticipation, actual) and type of intervention (CFI and control imagery) as the within-participants factors.

\section{Results}

\section{Physiological Indices of Well-being}

Descriptive statistics of sAA levels as a function of time and condition are presented in Table 1. Baseline sAA did not differ by condition $F(1,32)=1.93, p=0.17, \eta p^{2}=0.06$. An initial $2 \times 2 \times 2$ ANOVA that included intervention order as a between-subjects factor along with within-subjects factors, time and condition revealed that there were no interaction effects with intervention order $(p>0.1)$; in particular, the order $\times$ condition $\times$ time interaction was not significant $F(1$, $31)=0.18, p=0.64, \eta p^{2}=0.006$. Thus, the counterbalancing control that was employed was effective and subsequent analysis did not include interaction terms (Chin and Lee, 2008). Examination of sAA responses using ANCOVA with age as a covariate revealed a significant main effect of time, $\left.F(1,32)=6.13, p=0.02, \eta p^{2}=0.16\right)$, but this was qualified by a significant condition by time interaction $(F(1,32)=$ 9.62, $\left.p=0.004, \eta p^{2}=0.23\right)$; age was a significant covariate ( $p=0.004$; but an additional ANCOVA indicated that gender was not a significant covariate, $p=0.11)$. This condition by time interaction is depicted in Fig. 1 and reflects a stress response in the control imagery condition but minimal response in the CFI imagery, confirmed in post hoc analyses. Here, Bonferroni-corrected ANCOVA comparisons, with age as a covariate, revealed that in the control condition, participants exhibited increases in SAA responses as a consequence of actual pain compared with anticipation, suggestive of a stress response, $F(1,32)=9.72, p=0.004, \eta p^{2}=0.23$. In the CFI condition, there were no differences between anticipation and actual pain responses, $F(1,32)=0.81, p=0.38, \eta p^{2}=$ 0.02 . Additionally, there were no differences in anticipatory sAA responses between the control and CFI conditions. During cold pressor (i.e. actual pain), however, sAA

Table 1 Means (SDs) of physiological (sAA) responses by condition and time

\begin{tabular}{llll}
\hline & T1 (baseline) & T1 (Anticipation) & T3 (actual) \\
\hline CFI & $50.18(58.28)$ & $47.00(52.58)$ & $48.62(47.80)$ \\
Control & $44.58(37.09)$ & $50.84(51.04)$ & $56.38(49.73)$ \\
\hline
\end{tabular}

responses were higher in the control condition compared with the CFI condition, $F(1,32)=4.42, p=0.04, \eta p^{2}=0.12$.

\section{Pain Tolerance}

The initial Cox regression incorporating just condition (CFI, control) as a predictor of hazard produced no significant effects (hazard ratio $=0.78, p=0.42$ ), indicating that condition did not influence participants' pain tolerance; mean (SD) for $\mathrm{CFI}=195$ (116) seconds versus mean (SD) for control $=180$ (111) seconds.

To examine whether pain tolerance was influenced by participants' sAA anticipatory or actual responses to pain, we next incorporated sAA responses (i.e. delta scores) into the Cox regression model. The model using anticipatory sAA responses indicated a significant effect of anticipatory sAA responses on pain tolerance (hazard ratio $=1.04, p=0.02$ ), suggesting participants with larger anticipatory sAA responses were more likely to remove their hands from the water earlier. However, this model did not change the effect of condition (hazard ratio $=0.92, p=0.69$ ), and there was no interaction with condition (hazard ratio $=0.99, p=0.31$ ).

The model using actual sAA responses to pain also revealed a significant effect of actual sAA responses on pain tolerance (Hazard Ratio $=1.07, p=0.001$ ), although this effect was moderated by condition (Hazard Ratio $=0.96, p=$ 0.003). To expand, in the CFI condition, participants who exhibited larger actual sAA responses were more likely to remove their hands from the water earlier (Hazard Ratio = $1.04, p=0.003$ ), whereas actual sAA responses did not predict pain tolerance in the control imagery condition (Hazard Ratio $=0.99, p=0.45)$. This model did not change the effect of condition (Hazard Ratio $=0.66, p=0.23$ ).

\section{Emotional Indices of Well-being}

Descriptive statistics as a function of time and condition for each emotional index of well-being are presented in Table 2. Baseline measures did not differ by conditions $F(1,32)<1$, $p>0.1, \eta p^{2}<0.05$. MANOVA revealed no main effect of imagery condition $\left(F(8,26)=.53, p=0.83, \eta p^{2}=0.14\right)$, although a significant main effect of time on emotional measures was observed $\left(F(16,18)=3.19, p=0.01, \eta p^{2}=0.74\right)$. Univariate ANOVAs revealed changes in safe $(C)$, relaxed (C), safe (E), relaxed (E) and negative affect (again, see Table 2). Importantly, there was no interaction between time and imagery condition $\left(F(16,18)=.42, p=0.96, \eta p^{2}=0.27\right)$.

\section{Imagery Fidelity}

Comparisons were also made to establish if there were any differences in participants ability to engage with the CFI and control imagery interventions. Paired-samples t-test revealed 
Fig. 1 Salivary alpha-amylase delta score changes as a function of imagery (CFI, control) and pain (anticipation, actual) condition, with age covariate estimates. Bars represent one standard error of the mean (SEM)

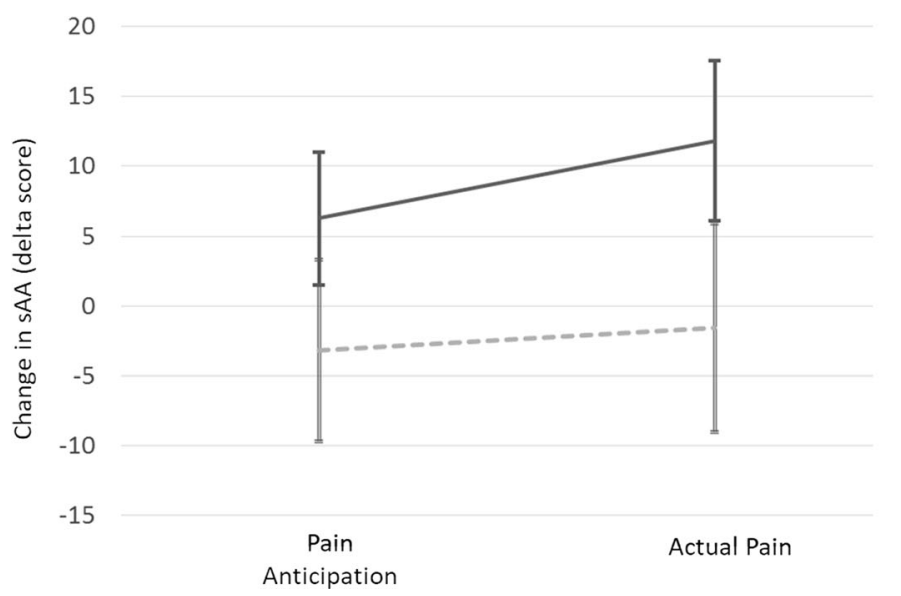

Control CFI
Table 2 Means (SDs) of emotional indices of well-being by condition and time

\begin{tabular}{|c|c|c|c|}
\hline & T1 (baseline) & T2 (anticipation) & T3 (actual) \\
\hline \multicolumn{4}{|c|}{ TPAS safe $(\mathrm{C})^{*}$} \\
\hline CFI & $12.38(2.67)$ & $13.15(2.41)$ & $11.85(2.98)$ \\
\hline Control & $11.79(3.48)$ & $13.12(2.51)$ & $11.56(3.16)$ \\
\hline \multicolumn{4}{|c|}{ TPAS relaxed $(\mathrm{C})^{*}$} \\
\hline CFI & $10.79(3.01)$ & $12.06(2.68)$ & $10.47(2.85)$ \\
\hline Control & $10.85(2.69)$ & $12.44(2.77)$ & $10.56(3.07)$ \\
\hline \multicolumn{4}{|c|}{ TPAS active (C) } \\
\hline CFI & $10.32(3.84)$ & $9.71(3.63)$ & $10.21(3.76)$ \\
\hline Control & $10.06(3.66)$ & $9.62(3.91)$ & $9.97(4.00)$ \\
\hline \multicolumn{4}{|c|}{ TPAS safe $(\mathrm{E})^{*}$} \\
\hline CFI & $13.35(2.51)$ & $13.91(2.84)$ & $12.91(3.51)$ \\
\hline Control & $13.38(2.86)$ & $14.00(2.45)$ & $12.71(3.63)$ \\
\hline \multicolumn{4}{|c|}{ TPAS relaxed $(\mathrm{E}) *$} \\
\hline CFI & $12.94(1.93)$ & $13.74(2.26)$ & $12.62(3.26)$ \\
\hline Control & $13.79(2.37)$ & $12.59(3.07)$ & $12.09(3.37)$ \\
\hline \multicolumn{4}{|c|}{ TPAS active (E) } \\
\hline CFI & $12.09(3.37)$ & $11.47(3.49)$ & $11.59(3.63)$ \\
\hline Control & $11.74(3.74)$ & $12.00(3.52)$ & $11.82(3.55)$ \\
\hline \multicolumn{4}{|c|}{ PANAS (P) } \\
\hline CFI & $27.71(9.30)$ & $25.79(9.61)$ & $28.15(9.70)$ \\
\hline Control & $28.35(7.10)$ & $26.76(8.84)$ & $27.65(9.48)$ \\
\hline \multicolumn{4}{|c|}{ PANAS $(\mathrm{N}) *$} \\
\hline CFI & $11.68(1.72)$ & $10.56(1.08)$ & $12.03(3.38)$ \\
\hline Control & $11.79(3.36)$ & $10.71(1.45)$ & $12.09(2.83)$ \\
\hline
\end{tabular}

Key: $C$, characteristic; $E$, enjoyment; $P$, positive; $N$, negative

*Significant effect of time no differences for ease of experiencing the imagery, resistance towards the imagery, how hard it was to create an image, including how clearly it could be seen and, finally, how tense they felt at that present moment; $t(33)<1.8, p>0.09$ in all cases (see Table 3 ). In a final exploratory analysis, the imagery fidelity items were correlated with pain tolerance. Notably, only ease of imagery was significant. The easier participants found the respective imagery task, the longer they kept their hand submerged in the cold pressor during that condition ( $r=$ $0.37, p=0.03$ for CFI, and $r=0.31, p=0.08$ for control).

\section{Discussion}

The purpose of the present study was to investigate the impact of CFI on pain coping, including when subjected to actual pain and when in anticipation of this pain. In respect to actual pain, we found that CFI, as compared with control imagery, attenuated sAA levels to pain. However, whilst pain tolerance did not differ by imagery condition (CFI, control), sAA levels to actual pain predicted decreased pain tolerance in the $\mathrm{CFI}$ condition. We further found that anticipatory sAA responses were predictive of pain tolerance, but that this effect was not moderated by condition. To expand, participants with larger anticipatory sAA responses were more likely to remove their hands from the water earlier. Of note, none of the emotional measures of well-being differed by imagery condition, nor by condition over time. Thus, our results indicate effects of CFI

Table 3 Means (SDs) of imagery fidelity by condition

\begin{tabular}{lll}
\hline & CFI & Control \\
\hline 1. Ease of experiencing the imagery & $4.59(3.00)$ & $4.12(2.80)$ \\
2. Resistance towards the imagery & $4.12(2.85)$ & $4.92(3.07)$ \\
3. How hard it was to create an image & $4.32(2.43)$ & $4.94(2.26)$ \\
4. How clearly it could be seen & $7.91(1.98)$ & $7.76(2.43)$ \\
5. How tense they felt at that present moment & $4.88(3.18)$ & $4.59(2.73)$ \\
\hline
\end{tabular}


on physiological and behavioural measures of pain coping, but not psychological measures of pain coping.

A main result of the present study was that CFI attenuated the stress response to acute pain. That is, participants demonstrated no increase in sAA responses whilst subjected to cold pressor pain, when engaging with CFI, but when the same participants engaged with a control (nature) imagery whilst subjected to cold pressor pain, sAA increases were observed. This result is similar to the findings of Arch et al. (2014) who also observed blunting of sAA levels when participants were subjected to psychological stress, following compassion training. They argue that lower sAA responses can be interpreted as dampened sympathetic nervous system activity (see also Bauer et al. 2002) and concluded that self-compassion training can diminish psychobiological responses to threat (in their case the threat of social evaluation). As sAA levels are a reliable marker of psychological stress and fast-acting sympathetic nervous system responding (Nater and Rohleder 2009), our results demonstrate that engaging with CFI provided downregulation of rapid autonomic nervous system responses to stress when experiencing acute pain. As we only observed these effects when participants engaged in the CFI, and not the control imagery, this demonstrates that our CFI intervention was effective in dampening physiological pain responses. That is, if simply distraction or attention were key, then we would not have observed time-by-condition interaction effects. To expand, we found no difference in our participants' ability to engage in the CFI and control imagery, including ease of experiencing the imagery, difficulty creating an image, its visualisation aspects and/or resistance towards the imagery. This indicates that it was the compassionate nature of the task, rather than cognitive load, that resulted in the dampening of the physiological sAA stress response. Relatedly, some recent research has found no effect of non-judgmental body scanning on pain responding (Sharpe et al. 2013). This is consistent with our argument that it is the affiliative emotional aspects of the imagery that is a critical factor in mediating pain responding.

Compassion-focused interventions and pain coping can both be linked to activation of the affiliative affect system, with previous psychological and behavioural research demonstrating that social support increases pain tolerance and psychological pain coping (Brown et al. 2003; Cano and Williams 2010; Gregory et al. 2017; Jackson et al. 2005). Additionally, Gooding et al. (2020) have argued that in individuals who experience persistent pain, the threat system may become dysregulated and compassion-based interventions address this dysregulation. In explaining our findings, it can be hypothesised that engaging in CFI activated the affiliative/ self-soothing system linked to parasympathetic nervous system activation, allowing for sympathetic nervous system regulation. Recently, oxytocin has been found to increase affiliative processes such as compassion, and intranasal administration of oxytocin increases pain tolerance to experimental noxious stimuli (Boll et al. 2018). We hence speculate that the blunted sAA responses observed reflect CFI: (i) providing a buffering effect in response to pain/pain perception and appraisal and/or (ii) downregulation of the stress response as a consequence of affiliative affect system activation. However, to further investigate mechanisms underlying exactly how CFI impacted upon the physiological stress response to acute pain, in future research, it is recommended that measures of oxytocin are also taken.

Contrary to predictions, we did not find an effect of condition (CFI vs. control) on pain tolerance; engaging in CFI did not significantly impact upon how long participants were able to keep their hand emerged in the cold water, as compared with control imagery. Whilst the study was powered to detect medium effects, the effects we observed were smaller. Therefore, larger studies are needed to detect similar effects (if they exist). This stated, in prior research, when effects of compassionbased approaches on pain tolerance have been found, they occur only in participants with a history of self-injury (Gregory et al. 2017). Hooley and St. Germain (2014) suggest that those without a history of self-injury are likely to have a reasonable level of pain sensitivity, in which case one would not expect changes in pain tolerance, even if changes in (state) compassion are observed. Our results accord with this by demonstrating that in our non-selected participants, CFI did not affect pain tolerance, but did affect the stress response to pain. In future research it may therefore be useful to obtain information regarding history of self-injury in potential populations. Alternatively, it could be that what is key to pain tolerance is distraction or cognitive load. For example, research has demonstrated that distraction via virtual reality reduces reports of pain (e.g. Hoffman et al. 2000; Kipping et al. 2012). Consistent with this, we did find that ease of engaging with the imagery tasks was positively correlated with pain tolerance in both conditions. Added to this, as analysis of our imagery fidelity metrics revealed no difference in the ability of individuals to engage with the two different imagery types, it could be that both were equally effective (or otherwise) in altering pain tolerance. To explore this further, a control 'no intervention' group would be necessary. Assuming a suitable sample size, this would allow one to establish if simple distraction is, or is not, key to behavioural indices of pain tolerance.

We did not find a direct effect of CFI on anticipatory sAA responses. However, we did observe that participants with larger anticipatory sAA responses per se, were more likely to remove their hands from the water earlier. Thus, a greater physiological sympathetic nervous system response to the anticipation of pain was associated with decreased pain tolerance (Kyle and McNeil, 2014). Pain anticipation and expectation is frequently related to pain tolerance (Burton et al. 2016; Peerdeman et al. 2016). Whilst we did not find an effect of CFI on anticipatory pain, it should be noted that in previous 
research where differences have been found (e.g. Harwood and Kocovski, 2017), the anxiety provoking scenario (a speech task), as well as the measure of anticipatory anxiety (Spielberger State-Trait Anxiety Inventory-State Version) were quite different. Additionally, Harwood and Kocovski only observed effects for high anxious participants. This suggests that a range of psychological and physiological measures be taken in future research to better explore the effects of CFI on anticipatory responses.

sAA responses were predictive of pain tolerance in the CFI condition, but not the neutral condition. To expand, participants who demonstrated larger sAA responses to painful stimuli when engaged in CFI were more likely to withdraw their hand from the cold water earlier. Previously, sAA levels have not been shown to impact upon behavioural indices of pain tolerance. That is, although Wittwer et al. (2016) found sAA levels to increase with subjective ratings of acute pain intensity and unpleasantness, they found no relationship between sAA and objective ratings of pain tolerance (in their research, this was increases in heat stimuli that an individual could tolerate). As we found sAA responses were predictive of behavioural pain tolerance, but only in the CFI condition, this suggests that there is a relationship between pain sensitivity, the physiological stress responses and compassion. Of note, it has been observed that individuals who demonstrate pain insensitivity are more likely to be self-critical. As self-criticism is diametrically opposed to compassion (Gilbert et al. 2004; Neff et al. 2019), it can be speculated that in our study individuals who did not show dampening of the sAA responses did not demonstrate the benefits of the CFI intervention and hence withdrew their hand from the water given the associated physiological stress response and 'painful' experience (Peckerman et al. 1994). However, participants in the current study were selected based on low self-criticism scores; post hoc, we examined relationships between FRCRS subscales, pain tolerance and sAA responses, but they were not related (these data are available from the authors). Gregory et al. (2017) have found that individuals with a history of self-injury, as compared with those without, demonstrated greater pain sensitivity (to cold pressor) following a task designed to increase self-compassion. As such, they suggest that increased self-compassion (in their study induced by a values affirming task) helped correct the pain insensitivity that is typically found in those who self-harm. In this context, an elevated physiological stress response and 'painful' experience would lead to greater sensitivity to pain through the CFI and consequently also explain the reduced pain tolerance for those whom demonstrated larger sAA responses. Future intervention studies should examine these possibilities with a sample with a range of self-criticism and self-compassion scores, and assess changes in state measures, to clarify these relationships.

Finally, whilst we observed a number of changes concerning the effects of CFI on our physiological indices of well-being, our emotional measures of well-being were not influenced by the different imagery conditions, nor by condition over time. Whilst Penlington (2019) observed changes in psychological indices of well-being (e.g. anxiety), the parameters of that research were considerably different; that is, the intervention lasted a period of 12 months. This highlights the importance of choosing brief measures that are sensitive to change alongside physiological measures that can be obtained easily (e.g. non-invasively). Additionally, whilst it is possible that participants were fatigued by the completion of the same measures, three times over the course of an hour, changes over time were observed in the expected directions. To expand, immediately before pain induction (i.e. anticipation of the painful cold pressor task), participants reported less feelings of safety and relaxation, and greater feelings of negative affect, as compared with baseline and post-pain induction. This suggests that participants were not fatigued by completion of the measures (e.g. acquiescence bias), but neither intervention influenced emotional responding either.

\section{Limitations and Future Research Directions}

It is important to note that despite our promising results, before clinical efficacy can be argued, our findings need replication in both larger, and clinical, samples. In those replications, individual differences and potential confounds, such as smoking and hormone status that we did not assess due to the repeated measures design, should be investigated or controlled. A second limitation of the present research was our failure to measure intensity of pain perception which, in previous studies of pain (i.e. self-injury) and compassion, has been found to be an important outcome variable. That is, in such research, compassion-based interventions have been found to robustly impact upon ratings of pain intensity if not pain tolerance (Gregory et al. 2017). In future research, therefore, a measure of pain intensity (and unpleasantness) would be a significant improvement to the present design. Thirdly, if CFI is attenuating physiological stress responses to pain via upregulation of the soothing/affiliative system, then we would also expect behavioural reports of self-compassion to increase following the specific CFI. Thus, it is of paramount importance that any future research investigating effects of CFI upon physiological, behavioural and psychological pain coping also includes a state measure of self-compassion. Additionally, our control imagery task may not have been as neutral as intended; recent work has highlighted that imagery and other ways of connecting to nature are stress reducing. For example, proximal nature buffers the effects of low levels of social connection on well-being (Cartwright et al. 2018). This stated, we did find our hypothesised physiological dampening for CFI relative to the nature imagery task, and participants reported similar imagery fidelity. 
Our intervention further promoted self-compassion, which is distinct from compassion for others (López et al. 2018). Interestingly, empathy to others' pain has been found to increase pain perception due to its relation to attachment processes including social rejection (Eisenberger 2012; MacDonald and Leary 2005). Thus, studies that investigate the interplay between self-compassion and compassion to others (Gilbert 2014) in relation to pain are warranted. Of relevance here, Carson et al. (2005) demonstrated that an 8week loving-kindness programme for chronic lower back pain patients significantly improved psychological coping. Their programme included elements of loving-kindness (including compassion) towards the self, as well as others-including loved ones, a neutral person and those they had experienced difficulties with, or who had previously harmed them. Results revealed that the loving-kindness meditation (LKM) reduced ratings of pain, anger, and psychological distress in the patient group. Carson et al. hence argued that LKM may be a useful therapy that clinicians can integrate into their pain treatment programmes, whilst also noting that the mechanisms via which it operates needs further exploration. Certainly, teasing apart the relative importance of different aspects of compassion (and LKM) is a valid future direction. This includes investigation of any mediating effects of whom the imagery is directed towards and/or from; and whether the CFI is imagined or embodied.

One strength of the present study was the use of a crossover repeated measures design. This allowed for substantial control over extraneous variables, such as time of day, which, at least in part, minimised baseline differences between variables of interest; and there were no apparent crossover effects. Indeed, the approach is often described as the gold standard of trials (Mills et al. 2009). However, it does limit the possibility of examining individual differences. Of rising importance here, is the inclusion of aphantasia (i.e. imagery visualisation ability) as a moderating variable. With the efficacy of imagery style interventions becoming increasingly investigated and reported, factoring in an individual's ability to engage with imagery per se is an important next step in the design of future studies. Certainly, in reviewing the fidelity data, that participant generally reported moderate ratings of their ability to engage with the imagery, including creating an image, indicates that for some engaging in the imagery tasks might not have been that easy. This of course could have also influenced findings (e.g. implementation efficacy).

To conclude, our data demonstrate that CFI provides a dampening of the physiological stress response to actual painful stimuli. To more comprehensively evaluate the effectiveness of CFI on pain coping and pain tolerance, it is important that previous history of self-injury, anxiety etc. is investigated alongside the actual intervention as well as self-reports of compassion and pain intensity/unpleasantness. Additionally, to more fully understand the physiological mechanisms via which
CFI is exerting its pain coping effects, a measure of affiliative responding (e.g. oxytocin) would be useful. Other compassionbased approaches, for example compassion for others, could also be evaluated in the context of pain to ascertain if it is the affiliative emotional content of the imagery that is key to effects observed. Indeed, as CFI appears to offer a means by which to dampen physiological stress responses to pain, it may in future offer new avenues for therapeutic interventions, including those for chronic pain patients (Purdie and Morley 2016).

Acknowledgements We thank Andrew Baird for his help in conceptualising the study, and Richard Steele/James Brown for their help with data collection. We further thank the four anonymous reviewers whose detailed feedback has much improved the manuscript.

Author Contributions FM and DS conceptualised and designed the study. FM and DS conducted the study. DS led on data analysis. FM led on manuscript preparation. FM and DS both edited and approved the final manuscript.

\section{Compliance with Ethical Standards}

Statement of Ethics All research procedures received University of Derby research ethics committee approval and were in accord with the BPS code of conduct and ethics (2018). This includes obtaining fully informed consent from all individuals who participated in the research.

Conflict of Interest FM reports grants from the Compassionate Mind Foundation, during the conduct of the study. Dr Maratos and Professor Sheffield state that there is no conflict of interest with respect to this research.

Open Access This article is licensed under a Creative Commons Attribution 4.0 International License, which permits use, sharing, adaptation, distribution and reproduction in any medium or format, as long as you give appropriate credit to the original author(s) and the source, provide a link to the Creative Commons licence, and indicate if changes were made. The images or other third party material in this article are included in the article's Creative Commons licence, unless indicated otherwise in a credit line to the material. If material is not included in the article's Creative Commons licence and your intended use is not permitted by statutory regulation or exceeds the permitted use, you will need to obtain permission directly from the copyright holder. To view a copy of this licence, visit http://creativecommons.org/licenses/by/4.0/.

\section{References}

Arch, J. J., Brown, K. W., Dean, D. J., Landy, L. N., Brown, K. D., \& Laudenslager, M. L. (2014). Self-compassion training modulates alpha-amylase, heart rate variability, and subjective responses to social evaluative threat in women. Psychoneuroendocrinology, 42, 49-58. https://doi.org/10.1016/j.psyneuen.2013.12.

Bauer, A. M., Quas, J. A., \& Boyce, W. T. (2002). Associations between physiological reactivity and children's behavior: Advantages of a multisystem approach. Journal of Developmental \& Behavioral Pediatrics, 23(2), 102-113 https://psycnet.apa.org/doi/10.1097/ 00004703-200204000-00007.

Boll, S., Almeida de Minas, A., Raftogianni, A. C., Herpertz, S. C., \& Grinevich, V. (2018). Oxytocin and pain perception: From animal 
models to human research. Neuroscience, 387, 149-161. https://doi. org/10.1016/j.neuroscience.2017.09.041.

Brown, J. L., Sheffield, D., Leary, M. R., \& Robinson, M. E. (2003). Social support and experimental pain. Psychosomatic Medicine, 65(2), 276-283. https://doi.org/10.1097/01.psy.0000030388. 62434.46 .

Burton, A. R., Fazalbhoy, A., \& Macefield, V. G. (2016). Sympathetic responses to noxious stimulation of muscle and skin. Frontiers in Neurology, 7, 109. https://doi.org/10.3389/fneur.2016.00109.

Cano, A., \& Williams, A. C. (2010). Social interaction in pain: Reinforcing pain behaviors or building intimacy? Pain, 149(1), 9 11. https://doi.org/10.1016/j.pain.2009.10.010.

Carson, J. W., Keefe, F. J., Lynch, T. R., Carson, K. M., Goli, V., Fras, A. M., \& Thorp, S. R. (2005). Loving-kindness meditation for chronic low back pain: Results from a pilot trial. Journal of Holistic Nursing, 23(3), 287-304. https://doi.org/10.1177/0898010105277651.

Cartwright, B., White, M., \& Clitherow, T. (2018). Nearby nature 'buffers' the effect of low social connectedness on adult subjective wellbeing over the last 7 days. International Journal of Environmental Research and Public Health, 15(6), 1238. https:// doi.org/10.3390/ijerph15061238.

Chin, R., \& Lee, B. Y. (2008). Principles and practice of clinical trial medicine. Elsevier.

Duarte, J., McEwan, K., Barnes, C., Gilbert, P., \& Maratos, F. A. (2015). Do therapeutic imagery practices affect physiological and emotional indicators of threat in high self-critics? Psychology and Psychotherapy: Theory, Research and Practice, 88(3), 270-284. https://doi.org/10.1111/papt.12043.

Depue, R. A., \& Morrone-Strupinsky, J. V. (2005). A neurobehavioral model of affiliative bonding: Implications for conceptualizing a human trait of affiliation. Behavioral and Brain Sciences, 28(3), 313 349.

Eisenberger, N. I. (2012). The pain of social disconnection: Examining the shared neural underpinnings of physical and social pain. Nature Reviews Neuroscience, 13(6), 421-434. https://doi.org/10.1038/ nrn3231.

Gilbert, P. (2014). The origins and nature of compassion focused therapy. British Journal of Clinical Psychology, 53(1), 6-41. https://doi.org/ 10.1111/bjc. 12043 .

Gilbert, P. (2017). A brief outline of the evolutionary approach for compassion focused therapy. EC Psychology and Psychiatry, 3(6), 218227.

Gilbert, P., Clarke, M., Hempel, S., Miles, J. N., \& Irons, C. (2004). Criticizing and reassuring oneself: An exploration of forms, styles and reasons in female students. British Journal of Clinical Psychology, 43(1), 31-50. https://doi.org/10.1348/ 014466504772812959.

Gilbert, P., McEwan, K., Mitra, R., Franks, L., Richter, A., \& Rockliff, H. (2008). Feeling safe and content: A specific affect regulation system? Relationship to depression, anxiety, stress, and self-criticism. The Journal of Positive Psychology, 3(3), 182-191. https://doi.org/ 10.1080/17439760801999461.

Gooding, H., Stedmon, J., \& Crix, D. (2020). All these things don't take the pain away but they do help you to accept it': Making the case for compassion-focused therapy in the management of persistent pain. British Journal of Pain, 14(1), 31-41. https://doi.org/10.1177/ 2049463719857099.

Gregory, W. E., Glazer, J. V., \& Berenson, K. R. (2017). Self-compassion, self-injury, and pain. Cognitive Therapy and Research, 41(5), 777-786 https://psycnet.apa.org/doi/10.1007/s10608-017-9846-9.

Harwood, E. M., \& Kocovski, N. L. (2017). Self-compassion induction reduces anticipatory anxiety among socially anxious students. Mindfulness, 8(6), 1544-1551. https://doi.org/10.1007/S12671017-0721-2.

Hoffman, H. G., Patterson, D. R., \& Carrougher, G. J. (2000). Use of virtual reality for adjunctive treatment of adult burn pain during physical therapy: A controlled study. The Clinical Journal of Pain, 16(3), 244-250. https://doi.org/10.1097/00002508-20000900000010 .

Hofmann, S. G., Grossman, P., \& Hinton, D. E. (2011). Loving-kindness and compassion meditation: Potential for psychological interventions. Clinical Psychology Review, 31(7), 1126-1132. https://doi. org/10.1016/j.cpr.2011.07.003.

Hooley, J. M., \& St. Germain, S. A. (2014). Nonsuicidal self-injury, pain, and self-criticism: Does changing self-worth change pain endurance in people who engage in self-injury? Clinical Psychological Science, 2(3), 297-305. https://doi.org/10.1177/ 2167702613509372

Jackson, T., Iezzi, T., Chen, H., Ebnet, S., \& Eglitis, K. (2005). Gender, interpersonal transactions, and the perception of pain: An experimental analysis. The Journal of Pain, 6(4), 228-236. https://doi. org/10.1016/j.jpain.2004.12.004.

Kim, J. J., Cunnington, R., \& Kirby, J. N. (2020a). The neurophysiological basis of compassion: An fMRI meta-analysis of compassion and its related neural processes. Neuroscience \& Biobehavioral Reviews, 108, 112-123 https://psycnet.apa.org/doi/10.1016/j.neubiorev. 2019.10.023.

Kim, J. J., Parker, S.1., Doty, J. R., Cunnington, R., Gilbert, P., \& Kirby, J. N. (2020b). Neurophysiological and behavioural markers of compassionate motivation. Nature Research, 10, 6789. https://doi.org/ 10.1038/s41598-020-63846-3.

Kipping, B., Rodger, S., Miller, K., \& Kimble, R. M. (2012). Virtual reality for acute pain reduction in adolescents undergoing burn wound care: A prospective randomized controlled trial. Burns, 38(5), 650-657. https://doi.org/10.1016/j.burns.2011.11.010.

Kirby, J. N., Doty, J. R., Petrocchi, N., \& Gilbert, P. (2017a). The current and future role of heart rate variability for assessing and training compassion. Frontiers in Public Health, 5, 40. https://doi.org/10. 3389/fpubh.2017.00040.

Kirby, J. N., Tellegen, C. L., \& Steindl, S. R. (2017b). A meta-analysis of compassion-based interventions: Current state of knowledge and future directions. Behavior Therapy, 48(6), 778-792. https://doi. org/10.1016/j.beth.2017.06.003.

Kyle, B. N., \& McNeil, D. W. (2014). Autonomic arousal and experimentally induced pain: A critical review of the literature. Pain Research and Management, 19(3), 159-167. https://doi.org/10. $1155 / 2014 / 536859$.

Longe, O., Maratos, F. A., Gilbert, P., Evans, G., Volker, F., Rockliff, H., \& Rippon, G. (2010). Having a word with yourself: Neural correlates of self-criticism and self-reassurance. NeuroImage, 49(2), 1849-1856. https://doi.org/10.1016/j.neuroimage.2009.09.019.

López, A., Sanderman, R., Ranchor, A. V., \& Schroevers, M. J. (2018). Compassion for others and self-compassion: Levels, correlates, and relationship with psychological well-being. Mindfulness, 9(1), 325 331. https://doi.org/10.1007/s12671-017-0777-z.

MacDonald, G., \& Leary, M. R. (2005). Why does social exclusion hurt? The relationship between social and physical pain. Psychological Bulletin, 131(2), 202-223 https://psycnet.apa.org/doi/10.1037/ 0033-2909.131.2.202.

Maratos, F. A., Duarte, J., Barnes, C., McEwan, K., Sheffield, D., \& Gilbert, P. (2017). The physiological and emotional effects of touch: Assessing a hand-massage intervention with high self-critics. Psychiatry Research, 250, 221-227. https://doi.org/10.1016/j. psychres.2017.01.066.

Maratos, F. A., Montague, J., Ashra, H., Welford, M., Wood, W., Barnes, C., Sheffield, D., \& Gilbert, P. (2019). Evaluation of a compassionate mind training intervention with school teachers and support staff. Mindfulness, 10(11), 2245-2258. https://doi.org/10.1007/s12671019-01185-9.

Maratos, F. A., \& Pessoa, L. (2019). What drives prioritized visual processing? A motivational relevance account. Progress in Brain 
Research, 247, 111-148. https://doi.org/10.1016/bs.pbr.2019.03. 028.

Master, S. L., Eisenberger, N. I., Taylor, S. E., Naliboff, B. D., Shirinyan, D., \& Lieberman, M. D. (2009). A picture's worth: Partner photographs reduce experimentally induced pain. Psychological Science, 20(11), 1316-1318 https://psycnet.apa.org/doi/10.1111/j.14679280.2009.02444.x.

Mills, E. J., Chan, A.-W., Wu, P., Vail, A., Guyatt, G. H., \& Altman, D. G. (2009). Design, analysis and presentation of crossover trials. Trials, 10, 27. https://doi.org/10.1186/1745-6215-10-27.

Mitchell, L. A., MacDonald, R. A., \& Brodie, E. E. (2004). Temperature and the cold pressor test. The Journal of Pain, 5(4), 233-237. https:// doi.org/10.1016/j.jpain.2004.03.004.

Nater, U. M., Rohleder, N., Schlotz, W., Ehlert, U., \& Kirschbaum, C. (2007). Determinants of the diurnal course of salivary alpha-amylase. Psychoneuroendocrinology, 32(4), 392-401. https://doi.org/ 10.1016/j.psyneuen.2007.02.007.

Nater, U. M., \& Rohleder, N. (2009). Salivary alpha-amylase as a noninvasive biomarker for the sympathetic nervous system: Current state of research. Psychoneuroendocrinology, 34(4), 486-496. https://doi.org/10.1016/j.psyneuen.2009.01.014.

Neff, K. D., Tóth-Király, I., Yarnell, L. M., Arimitsu, K., Castilho, P., Ghorbani, N., et al. (2019). Examining the factor structure of the Self-Compassion Scale in 20 diverse samples: Support for use of a total score and six subscale scores. Psychological Assessment, 31(1), 27-45. https://doi.org/10.1037/pas0000629.

Peckerman, A., Hurwitz, B. E., Saab, P. G., Llabre, M. M., McCABE, P. M., \& Schneiderman, N. (1994). Stimulus dimensions of the cold pressor test and the associated patterns of cardiovascular response. Psychophysiology, 31(3), 282-290 https://psycnet.apa.org/doi/10. 1111/j.1469-8986.1994.tb02217.x.

Peerdeman, K. J., van Laarhoven, A. I., Peters, M. L., \& Evers, A. W. (2016). An integrative review of the influence of expectancies on pain. Frontiers in Psychology, 7, 1270. https://doi.org/10.3389/ fpsyg.2016.01270.

Penlington, C. (2019). Exploring a compassion-focused intervention for persistent pain in a group setting. British Journal of Pain, 13(1), 5966. https://doi.org/10.1177/2049463718772148.

Purdie, F., \& Morley, S. (2016). Compassion and chronic pain. Pain, 157(12), 2625-2627. https://doi.org/10.1097/j.pain. 0000000000000638 .

Rockliff, H., Gilbert, P., McEwan, K., Lightman, S., \& Glover, D. (2008). A pilot exploration of heart rate variability and salivary cortisol responses to compassion-focused imagery. Clinical Neuropsychiatry: Journal of Treatment Evaluation., 5(3), 32-139.

Rockliff, H., Karl, A., McEwan, K., Gilbert, J., Matos, M., \& Gilbert, P. (2011). Effects of intranasal oxytocin on 'compassion focused imagery'. Emotion, 11(6), 1388-1396 https://psycnet.apa.org/doi/10. 1037/a0023861.
Rohleder, N., \& Nater, U. M. (2009). Determinants of salivary $\alpha$-amylase in humans and methodological considerations. Psychoneuroendocrinology, 34(4), 469-485. https://doi.org/10. 1016/j.psyneuen.2008.12.004.

Sapolsky, R. M., Krey, L. C., \& McEwen, B. S. (1986). The neuroendocrinology of stress and aging: The glucocorticoid cascade hypothesis. Endocrine Reviews, 7(3), 284-301. https://doi.org/10.1210/ edrv-7-3-284.

Serafini, K., Malin-Mayor, B., Nich, C., Hunkele, K., \& Carroll, K. M. (2016). Psychometric properties of the Positive and Negative Affect Schedule (PANAS) in a heterogeneous sample of substance users. The American Journal of Drug and Alcohol Abuse, 42(2), 203-212. https://doi.org/10.3109/00952990.2015.1133632.

Sharpe, L., Nicholson Perry, K., Rogers, P., Refshauge, K., \& Nicholas, M. K. (2013). A comparison of the effect of mindfulness and relaxation on responses to acute experimental pain. European Journal of Pain, 17(5), 742-752. https://doi.org/10.1002/j.1532-2149.2012. 00241.x.

Singer, T., \& Bolz, M. (2013). Compassion: Bridging practice and science. Max Planck Institute for Human Cognitive and Brain Sciences.

Smith, K. E., \& Norman, G. J. (2017). Brief relaxation training is not sufficient to alter tolerance to experimental pain in novices. PLoS One, 12(5), e0177228. https://doi.org/10.1371/journal.pone. 0177228.

van Stegeren, A., Rohleder, N., Everaerd, W., \& Wolf, O. T. (2006). Salivary alpha amylase as marker for adrenergic activity during stress: Effect of betablockade. Psychoneuroendocrinology, 31(1), 137-141. https://doi.org/10.1016/j.psyneuen.2005.05.012.

Watson, D., Clark, L. A., \& Tellegen, A. (1988). Development and validation of brief measures of positive and negative affect: The PANAS scales. Journal of Personality and Social Psychology, 54(6), 1063-1070 https://psycnet.apa.org/doi/10.1037/0022-3514. 54.6.1063.

Wittwer, A., Krummenacher, P., La Marca, R., Ehlert, U., \& Folkers, G. (2016). Salivary alpha-amylase correlates with subjective heat pain perception. Pain Medicine, 17(6), 1131-1136. https://doi.org/10. 1093/pm/pnv085.

Wren, A. A., Somers, T. J., Wright, M. A., Goetz, M. C., Leary, M. R., Fras, A. M., et al. (2012). Self-compassion in patients with persistent musculoskeletal pain: Relationship of self-compassion to adjustment to persistent pain. Journal of Pain and Symptom Management, 43(4), 759-770. https://doi.org/10.1016/j. jpainsymman.2011.04.014.

Publisher's Note Springer Nature remains neutral with regard to jurisdictional claims in published maps and institutional affiliations. 